\title{
Adaptación en el manejo de la patología espinal en momentos de crisis sanitaria
}

\author{
Adaptation in the management of spinal pathology in times of sanitary crisis
}

\author{
Noel Alonso Calleros-Hernández, ${ }^{*}$ FJ Cruz-López, ${ }^{\ddagger} \mathrm{J}$ Ramos-Fernández-Villanueva, ${ }^{\ddagger}$ \\ JA De Haro-Estrada, ${ }^{\ddagger}$ Francisco Javier Sánchez-García, ${ }^{\ddagger}$ Alejandro Tejera Morett, ${ }^{\ddagger}$ \\ María Gabriela Moya-Arteta, ${ }^{*}$ Fernando Sosa-Sáenz, ${ }^{*}$ \\ Herman Michael Dittmar-Johnson ${ }^{\ddagger}$ \\ * Fellow Cirugía de Columna, Re-espalda A.C. \\ ${ }^{\ddagger}$ Cirujano de Columna, Re-espalda A.C. \\ Centro Médico Puerta de Hierro, Zapopan, Jalisco.
}

Correspondencia: Herman Michael Dittmar-Johnson. Boulevard puerta de Hierro No. 5150,

Torre C planta baja, Col. Puerta de Hierro, 45116, Zapopan, Jalisco. Correo electrónico: dittmar@cdcolumna.com

\section{RESUMEN}

Frente a la crisis sanitaria ocasionada por COVID-19, como cirujanos de columna realizamos modificaciones en nuestra práctica profesional con el objetivo de disminuir el riesgo de contagio de nuestro personal de salud, personal administrativo y de los pacientes. Con estos ajustes, también buscamos optimizar los recursos disponibles en las instituciones sanitarias y disminuir los riesgos perioperatorios en aquellos casos en los que postergar la intervención quirúrgica no es una opción. Por esta razón es necesaria la correcta identificación de los pacientes que ameritan una forzosa atención quirúrgica a corto plazo. Enumeramos una serie de recomendaciones para la atención de pacientes con patología espinal, basadas en una revisión y análisis de la bibliografía disponible para la atención en consulta y los protocolos de atención quirúrgica durante esta pandemia, tomando en cuenta que deben ajustarse respecto a la fase epidemiológica y a los recursos de cada entidad. Se ha realizado una clasificación de las patologías quirúrgicas dividiendo a los pacientes en cuatro categorías: emergencia, urgencia, semielectivas o electivas. Es nece-

\section{ABSTRACT}

Given the health crisis that resulted from the outbreak of the COVID-19, we as spine surgeons have made adjustments in our professional practice to lower the risk of infections of our healthcare workers, administrative personnel, and patients themselves. With these adjustments, we also aimed to optimize the use of available resources in health institutions and reduce preoperative risks in cases in which postponing a surgery is not an option. For this reason, it is of utmost importance to correctly identify patients that require emergent surgical intervention. After a thorough review and analysis of the available literature for consultation and surgical care protocols during this pandemic, we listed a series of recommendations for patient care with spinal pathology. It must be considered that these should be adjusted concerning the epidemiological phase and the resources of each entity. We created a classification of surgical pathologies to aid dividing patients into four categories: emergency, urgent, semi-elective, and elective. We consider it necessary to emphasize that these 
sario puntualizar que estas recomendaciones son una guía flexible y el tratamiento debe estar basado en esta información, además de las necesidades individuales del paciente así como en el juicio del cirujano, puesto que un grupo limitado de pacientes neurológicamente estables con enfermedad medular pueden deteriorarse con el pase del tiempo en el transcurso de esta contingencia, ameritando posteriormente un tratamiento de emergencia.

Palabras clave: COVID-19, cirugía de columna, manejo de patología espinal, triage. recommendations are a flexible guide. In addition to the given suggestions, treatments should be based on the patient's individual needs, as well as the surgeon's judgement, since a limited group of neurologically stable patients with spinal cord disease may eventually deteriorate during the course of this contingency, warranting emergency treatment afterwards.

Keywords: COVID-19, spine surgery, spine disease management, triage.

\section{INTRODUCCIÓN}

Frente a la crisis sanitaria ocasionada por COVID-19 y en atención a las recomendaciones de las autoridades sanitarias locales e internacionales, realizamos modificaciones en nuestra práctica profesional con el objetivo de disminuir el riesgo de contagio de nuestro personal de salud, personal administrativo y de los pacientes así como optimizar los recursos disponibles en las instituciones sanitarias y disminuir los riesgos perioperatorios en los casos que no es posible postergar una intervención quirúrgica. Por esta razón es necesaria la correcta identificación de los pacientes que ameritan una forzosa atención quirúrgica a corto plazo, difiriendo momentáneamente la cirugía electiva en pacientes cuyo padecimiento no condicione un riesgo potencial para la función a mediano o largo plazo., ${ }^{1,2}$

Por ello realizamos una revisión y análisis de la bibliografía sobre las recomendaciones y protocolos de atención quirúrgica durante esta pandemia, tomando en cuenta que ante esta situación los protocolos de atención cambian rápidamente a medida que avanza el estudio de esta enfermedad y deben ajustarse respecto a la fase epidemiológica y los recursos de cada país y de cada entidad en específico.

En relación a la patología espinal, se ha realizado una clasificación dividiendo a los pacientes en cuatro categorías que sirven como directriz en la toma de decisiones al momento de programar procedimientos quirúrgicos dividiéndolos en patologías de emergencia, urgencia, semielectivas o electivas (Tabla 1). ${ }^{3}$

\section{MATERIAL Y MÉTODOS}

Se realizó una revisión bibliográfica y análisis de la literatura disponible, respecto a artículos y guías para la prevención de la transmisión de COVID-19 y el manejo de patología espinal durante la contingencia sanitaria.

\section{DISCUSIÓN}

Ante las recomendaciones globales que se han generado en los últimos días en los distintos centros de cirugía de columna, utilizamos la clasificación antes men- 
cionada, dando prioridad a los pacientes con patología espinal de emergencia; sin embargo, consideramos necesario puntualizar que existen casos de pacientes neurológicamente estables con enfermedad medular que podrían deteriorarse con el paso del tiempo en el transcurso de esta contingencia. Queda a criterio del cirujano valorar la intervención a la brevedad debido que la resolución de este problema es incierta y en un futuro mediato los recursos sanitarios podrían limitarse exclusivamente para cirugía de emergencia, con el subsecuente deterioro funcional de pacientes a los que se pudo haber ofrecido resolución quirúrgica de forma temprana. ${ }^{2}$

Es importante destacar que, en los casos que se considera la posibilidad de una cirugía por dolor incapacitante o intratable con medicación ambulatoria, es posible realizar un bloqueo espinal terapéutico como medida que permita el control del dolor de forma temporal, permitiendo posponer la intervención quirúrgica durante el periodo de contingencia. ${ }^{3}$

Por otro lado, para reducir el riesgo de contagio, nuestro equipo recomienda adaptar las siguientes modificaciones en la práctica diaria:

- En la práctica de consulta, decidimos contactar a los pacientes previo a su cita, para identificar los casos en que no es posible postergar su consulta, sugiriendo asistir a consulta únicamente el paciente o con un solo familiar en caso necesario; se hace hincapié en la puntualidad a la consulta, debido que sólo se permite la entrada a la sala de espera a un paciente a la vez, al resto de los pacientes se les ha ofrecido la posibilidad de realizar una consulta de telemedicina o reprogramar la consulta. ${ }^{4}$

Tabla 1: Triage de pacientes con patología espinal.

\begin{tabular}{|c|c|c|}
\hline Triage & Definición & Patología \\
\hline Emergencia & Requiere cirugía en las primeras ocho horas & $\begin{array}{l}\text { 1. Síndrome de cauda equina } \\
\text { 2. Fractura con lesión medular incompleta }\end{array}$ \\
\hline Urgencia & Requiere cirugía en las primeras 48 horas & $\begin{array}{l}\text { 1. Déficit motor agudo o progresivo, inde- } \\
\text { pendientemente de la etiología } \\
\text { 2. Absceso epidural } \\
\text { 3. Dolor incapacitante con limitación de la } \\
\text { función, sin mejoría con TxCo } \\
\text { 4. Fístula de LCR sin mejoría con TxCo } \\
\text { 5. Fractura inestable }\end{array}$ \\
\hline Semielectiva & $\begin{array}{l}\text { Requiere cirugía en los primeros siete a } \\
14 \text { días }\end{array}$ & $\begin{array}{l}\text { 1. Fractura por osteoporosis o metastásica con } \\
\text { dolor incapacitante, sin mejoría con TxCo } \\
\text { 2. Infección que no mejora con TxCo } \\
\text { 3. Mielopatía cervical o dorsal progresiva }\end{array}$ \\
\hline Electiva & $\begin{array}{l}\text { La cirugía puede realizarse en seis a ocho } \\
\text { semanas }\end{array}$ & $\begin{array}{l}\text { 1. Todas las demás, independientemente de } \\
\text { su etiología. Estas cirugías se posponen }\end{array}$ \\
\hline
\end{tabular}


- En relación a la dinámica de consulta, se respeta cuidadosamente la distancia de seguridad interpersonal, teniendo contacto con el paciente exclusivamente durante la exploración física, previo y posterior a ésta se realiza el protocolo de desinfección de manos, por último, en caso de identificar casos sospechosos en el contexto de pacientes con síntomas respiratorios y/o fiebre, se les recomienda medicación sintomática y reposo en casa, también se les explica que sólo ante la presencia de datos de alarma es necesario acudir al servicio de urgencias para determinar si es necesaria la atención intrahospitalaria. ${ }^{5}$

La oficina permanece cerrada los siete días de la semana y el personal administrativo labora desde su hogar, asistiendo en caso necesario de forma escalonada. ${ }^{4}$

Respecto a los protocolos en quirófano, ante la presencia de pacientes con sospecha o confirmación de infección por COVID-19, modificamos las medidas de protección para disminuir riesgos al paciente y al equipo quirúrgico.

- Se recomienda bloqueo epidural en la medida de lo posible, situación poco común en cirugía espinal, en los casos que se realice intubación orotraqueal, es necesario considerar el uso de protocolo de intubación rápida sin ventilación previa para evitar la aerolización y dispersión del virus en la sala de operaciones. ${ }^{5,6}$

- Se recomienda optar por procedimientos de vía posterior de mínima invasión y evitar realizar procedimientos transorales. ${ }^{5}$

- El equipo y los bultos quirúrgicos se abren 20 minutos posterior al cierre del circuito aéreo del paciente. ${ }^{6}$

Durante el procedimiento quirúrgico recomendamos incluir exclusivamente al personal mínimo indispensable, el uso de tres pares de guantes, una bata impermeable y una bata quirúrgica estéril, cubrebocas N95 y máscara facial. ${ }^{4}$

Al término del procedimiento, recomendamos el retiro del primer par de guantes, aplicación de gel antibacterial, posteriormente el retiro del resto de equipo de protección (batas, máscara facial, cubrebocas), por último, el tercer par de guantes. ${ }^{4}$

\section{CONCLUSIÓN}

Es necesario puntualizar que estas recomendaciones son una guía flexible que permite orientar al cirujano de columna en la toma de decisiones al momento de realizar una cirugía durante esta contingencia sanitaria, así como respecto a las medidas que se deben llevar a cabo para disminuir el riesgo de contagio de su equipo de trabajo. Sin embargo, el tratamiento debe ser individualizado con base en esta información y en las necesidades individuales de cada paciente así como en el juicio del cirujano y el propio paciente, puesto que existe un grupo limitado de pacientes neurológicamente estables con enfermedad medular que puede deteriorarse con el paso del tiempo en el transcurso de la contingencia, ameritando 


\section{posteriormente tratamiento de emergencia en medio de un panorama epidémico menos favorable.}

\section{BIBLIOGRAFÍA}

1. Guy DK, Bosco JA III, Savoie FH III. AAOS. 31/03/2020. AAOS Guidelines on elective surgery during the COVID-19 Pandemic: March 31. Available from: https://aaos.org/globalassets/about/covid-19/aaos-guidelinneson-elective-surgery.pdf.

2. North American Spine Society. COVID-10 resources and updates. NASS [Internet]. 2020 [Cited 7 April 2020]; Available from: https://www.spine.org/Portals/0/assets/downloads/Publications/NASSInsider/ NASSGuidanceDocument040320.pdf

3. Sauri JC, Callejas E, García CL, Robles E. Recomendaciones para la toma de decisiones para realizar una cirugía de columna durante la pandemia de COVID-19, AMCICO 2020. Available from: http://www. smo.edu.mx/pdf/Recomendacion_AMCICO_covid-19.pdf.

4. Berjano P. Lessons from Milan: Spine care and COVID-19 [Internet]. Spinal News International. 2020 [Cited 6 April 2020]. Available from: https://spinalnewsinternational.com/lessons-from-milanspine-care-and-covid-19/.

5. Zou J, Yu H, Song D, Niu J, Yang H. Advice on standardized diagnosis and treatment for spinal diseases during the coronavirus disease 2019 pandemic. Asian Spine J. 2020; 14 (2): 258-263. doi: 10.31616/ asj.2020.0122.

6. Ti LK, Ang LS, Foong TW, et al. What we do when a COVID-19 patient needs an operation: operating room preparation and guidance. Can J Anesth/J Can Anesth. 2020; 67: 756-758. https://doi.org/10.1007/ s12630-020-01617-4.

\section{Código ORCID:}

Calleros-Hernández Noel Alonso https://orcid.org/0000-0001-5581-2821.

Sánchez-García Francisco Javier https://orcid.org/0000-0003-2033-9688.

Tejera Morett Alejandro https://orcid.org/0000-0003-4214-6254.

Moya-Arteta María Gabriela https://orcid.org/0000-0001-8668-0745.

Sosa-Sáenz Fernando https://orcid.org/0000-0003-0490-5053.

Dittmar-Johnson Herman Michael https://orcid.org/0000-0002-8024-7032. 